EGU21-9224, updated on 17 Dec 2021

https://doi.org/10.5194/egusphere-egu21-9224

EGU General Assembly 2021

(c) Author(s) 2021. This work is distributed under

the Creative Commons Attribution 4.0 License.

\title{
Analysis of space-based observations of peroxyacetyl nitrate (PAN) and its relation to other atmospheric tracers
}

\author{
Bruno Franco ${ }^{1}$, Lieven Clarisse ${ }^{1}$, Cathy Clerbaux ${ }^{1,2}$, and Pierre-François Coheur ${ }^{1}$ \\ ${ }^{1}$ Université Libre de Bruxelles (ULB), Spectroscopy, Quantum Chemistry and Atmospheric Remote Sensing (SQUARES), \\ Brussels, Belgium (bfranco@ulb.ac.be) \\ ${ }^{2}$ LATMOS/IPSL, Sorbonne Université, UVSQ, CNRS, Paris, France
}

Peroxyacetyl nitrate $\left(\mathrm{CH}_{3} \mathrm{C}(\mathrm{O}) \mathrm{O}_{2} \mathrm{NO}_{2}\right.$; abbreviated as PAN) is the main tropospheric reservoir of nitrogen oxide radicals $\left(\mathrm{NO}_{x}\right)$ and contributes to redistributing $\mathrm{NO}_{x}$ from source to remote regions. Recently, PAN total columns have been retrieved from the radiance spectra recorded by IASI (Infrared Atmospheric Sounding Interferometer) onboard the Metop satellite platforms, using a neural network-based retrieval approach. The daily global distributions obtained from these measurements provide a comprehensive picture of PAN through the troposphere.

Here we exploit as a climatology the 13-year time series of global PAN measurements derived from the IASI/Metop-A observations (October 2007 - December 2020) to characterize the spatial distribution and seasonal variability of PAN abundance worldwide. In particular, continental areas within the tropics appear to be source regions of PAN throughout the year, whereas PAN at North Hemisphere mid- and high latitudes exhibits a more pronounced seasonal cycle and peaks during the boreal summer. Strong outflows of PAN are captured over the oceans, downwind of continental source regions such as Eastern Asia and Eastern US. This dataset also allows us to investigate the recent trends of atmospheric PAN abundance over the last 13 years, over both source and remote areas.

To better understand what drives the spatial distribution and variability of PAN, we analyze alongside the regional time series of PAN those of carbon monoxide (CO) from IASI/Metop-A, and of formaldehyde $(\mathrm{HCHO})$ and nitrogen dioxide $\left(\mathrm{NO}_{2}\right)$ from OMl/Aura (Ozone Monitoring Instrument). Locally, we find simultaneous enhancements of PAN and CO abundances, which in this case indicates that most PAN originates from fire-derived precursors. This mainly occurs over the typical biomass burning regions in the tropics. Overall, strong correlations are observed over source areas between PAN and $\mathrm{HCHO}$, which is used here as a tracer of tropospheric chemistry and of the presence of oxygenated volatile organic compounds (OVOCs), while there is no particular correlation with $\mathrm{NO}_{2}$. The preliminary results suggest that PAN distribution and seasonality is primarily driven by the availability in OVOCs, and hence in peroxyacetyl radical, and that a locally weak $\mathrm{NO}_{2}$ abundance does not prevent the formation of PAN. 